\title{
Multimodality Renal Failure in a Patient with OEIS Complex
}

\author{
Jonathan D. Santoro, MD ${ }^{1}$ Stephanie Chao, MD² \\ ${ }^{1}$ Department of Pediatrics, Stanford University School of Medicine, \\ Palo Alto, California \\ 2 Department of Surgery, Stanford University School of Medicine, Palo \\ Alto, California \\ ${ }^{3}$ Department of Urology, Children's National Health System, \\ Washington, District of Columbia \\ ${ }^{4}$ Division of Neonatology, Department of Pediatrics, Stanford \\ University School of Medicine, Palo Alto, California
}

Michael H. Hsieh, MD, PhD ${ }^{3}$ Henry C. Lee, MD ${ }^{4}$

Address for correspondence Jonathan D. Santoro, MD, MSc, 730

Am J Perinatol Rep 2015;5:e161-e164.
Abstract
Keywords
- OEIS
- renal injury
- genitourinary
- obstructive
nephropathy

Omphalocele-exstrophy of the bladder-imperforate anus-spinal defect (OEIS) complex is a rare constellation of clinical abnormalities with wide phenotypic presentation. We describe a case of a preterm neonate with OEIS complex with acute renal failure, and the challenges in diagnosis and management of this patient as renal failure can be a multifactorial process when encountered with this rare complex.
Cloacal exstrophy with manifestations of the omphaloceleexstrophy of the bladder-imperforate anus-spinal defect (OEIS) complex was first described by Littre in 1709. Although there is debate regarding defining cloacal exstrophy as part of the spectrum of bladder defects, the OEIS complex was defined as omphalocele, cloacal exstrophy, imperforate anus and spinal cord defects in $1978 .^{1}$ These patients also can suffer from renal malformations and other anatomic abnormalities. $^{2}$

OEIS complex occurs at a rate of 1 in 200,000 to 400,000 live births. ${ }^{3}$ Although previously fatal, improvements in neonatal and surgical care have resulted in survival rates of 83 to $100 \%$, but often with prominent morbidities. ${ }^{4}$ The severity of associated congenital abnormalities in OEIS complex is variable, which can lead to unpredictable functional outcomes that are often difficult to anticipate during the immediate postnatal period. ${ }^{5}$ Renal abnormalities associated with reflux and subsequent hydronephrosis are near universal in patients with OEIS but these too are variable in their phenotypic presentations.

\section{Case}

A genetically female infant, prenatally diagnosed with OEIS complex based on fetal ultrasound and MRI, was delivered by cesarean delivery at 36 weeks' gestation for breech presentation and late decelerations to a 21-year-old primigravida Hispanic female. The umbilical cord was traumatically separated at delivery, and was clamped with roughly $40 \mathrm{~mL}$ of blood loss. No resuscitation was required. Physical examination ( $\mathbf{- F i g}$. 1a, b) of the patient was notable for a nondistended abdomen, with partially ruptured omphalocele with what appeared to be bowel with an open end and likely a distal end. Stool was leaking from the end of one of these loops of bowel. There was a wide midline defect with covering over abdominal contents by cord membrane. Below the umbilical cord, there was significant cloacal exstrophy with the hemibladders positioned laterally. Imperforate anus was present, and the rectum was extruding from the cloacal defect caudally with stool coming from it. No spinal defects were appreciated. The hips were splayed laterally. Other systems were grossly unremarkable. received

January 27, 2015 accepted after revision April 20, 2015

published online

June 12, 2015
DOI http://dx.doi.org/ 10.1055/s-0035-1554799. ISSN 2157-7005.
Copyright $\odot 2015$ by Thieme Medical Publishers, Inc., 333 Seventh Avenue, New York, NY 10001, USA. Tel: +1(212) 584-4662.
License terms

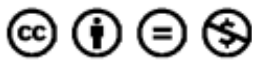



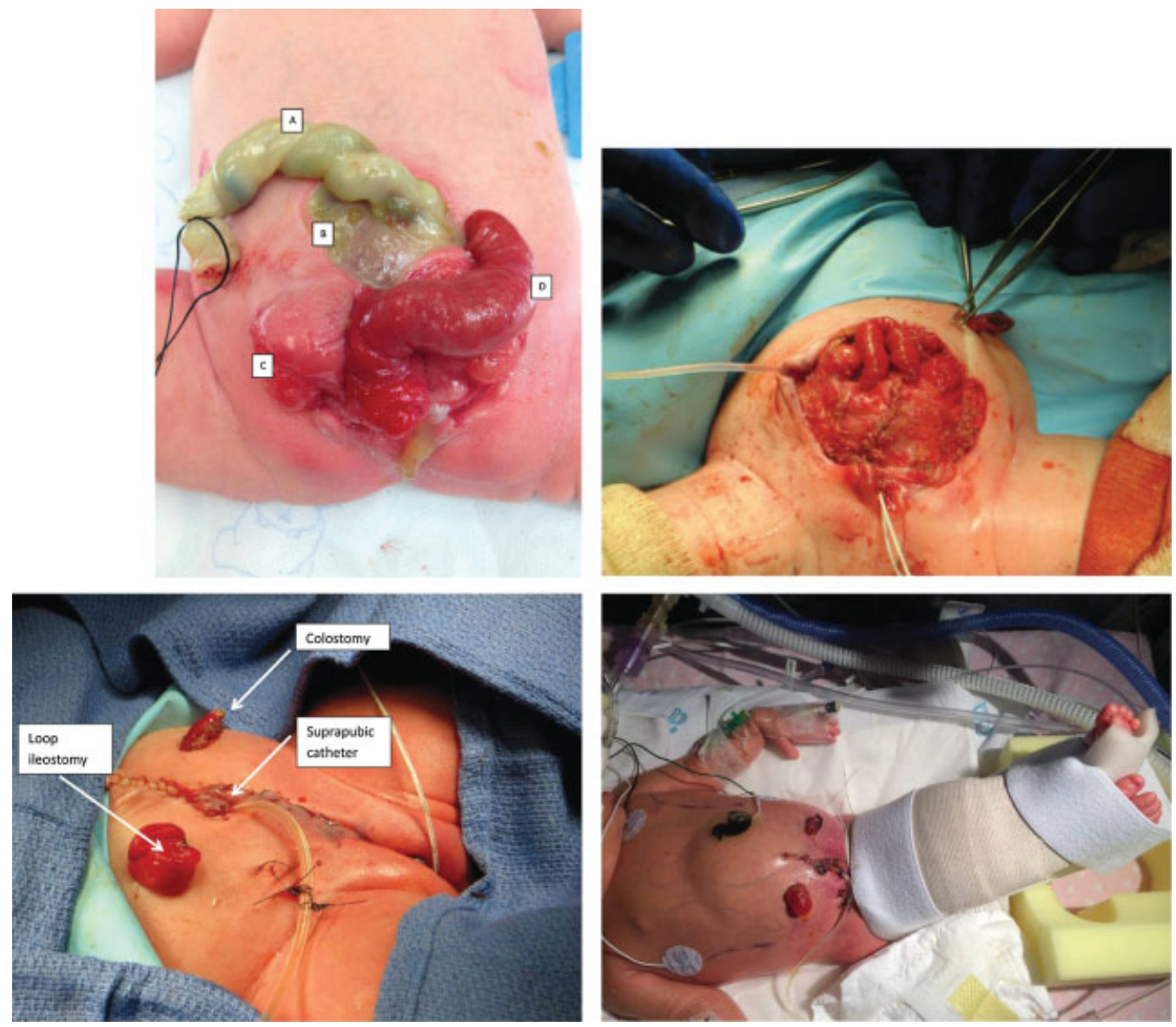

Fig. 1 (A) Initial presentation of patient at delivery. (a) Umbilical stump. (b) Omphalocele, (c) One half of hemibladder, and (d) Prolapsed rectum. (B) Postsurgical bladder closure. (C) Postoperative anatomy. (D) Postoperative "mermaid" wrap with postoperative changes visualized.

The pediatric surgery and urology teams determined that surgical repair should be performed expeditiously. A spinal ultrasound revealed a tethered cord which was determined to be inoperable at that time. Initial preoperative laboratories which included complete blood count, renal function panel, and venous blood gasses were unremarkable with the exception of a hematocrit of $39 \%$.

Preoperatively, the patient was hypotensive and tachycardic and was administered $10 \mathrm{~mL} / \mathrm{kg}$ of normal saline (NS) and transfused with packed red blood cells (pRBCs). On day of life 1 , the patient underwent closure repair of the cloacal exstrophy, with diverting loop ileostomy, colonic mucus fistula formation, pelvic closure, tubularization of the cecal plate, and abdominal wall closure via musculofascial flap, and bladder reconstruction. Following the procedure, the patient was left with one suprapubic catheter draining from the repaired bladder, two ureteral stents, a loop ileostomy on the right abdomen, and a diverted rectum exiting the left side of the abdomen (-Fig. 1c). Repair of imperforate anus was not performed. In addition, because of the pelvic closure, the patient was placed in a "mermaid" binder to prevent pelvic separation (-Fig. 1d).

In the 24 hours following her procedure, the patient was anuric. The patient received aggressive fluid resuscitation with NS and fresh frozen plasma (FFP) which increased urine output. Over the ensuing 4 days, the patient was noted to have uptrending blood urea nitrogen (BUN) and creatinine (Cr) (-Table 1). Repeat renal bladder ultrasounds demonstrated unchanged bilateral hydronephrosis without hydroureter and the patient's suprapubic catheter aspirated and flushed easily.

Table 1 Postoperative laboratory values for renal function

\begin{tabular}{|l|l|l|}
\hline Postoperative day & $\begin{array}{l}\text { Blood urea } \\
\text { nitrogen }(\mathrm{mg} / \mathrm{dL})\end{array}$ & $\begin{array}{l}\text { Creatinine } \\
\text { (mg/dL) }\end{array}$ \\
\hline 0 & 14 & 0.8 \\
\hline 1 & 19 & 1.2 \\
\hline 2 & 35 & 1.5 \\
\hline 3 & 44 & 1.8 \\
\hline 4 & 63 & 1.9 \\
\hline 5 (day of ascites tap) & 74 & 2.0 \\
\hline 6 & 71 & 1.9 \\
\hline 7 & 59 & 1.7 \\
\hline 14 & 20 & 0.8 \\
\hline 21 & 15 & 0.4 \\
\hline
\end{tabular}


On postoperative day 5 , the patient's girth enlarged from 28 to $37 \mathrm{~cm}$ in 24 hours. Abdominal ultrasound on day 6 demonstrated stable hydronephrosis but massive ascites were appreciated throughout the abdomen. A retrograde nephrostogram was performed urgently as there was concern that the rising levels of BUN and $\mathrm{Cr}$ may have indicated a leakage of urine upstream from the bladder as reabsorption of urine into the peritoneum may have caused this laboratory abnormality. This study revealed urine leak consistent with urinoma although a forniceal rupture secondary to retrograde pyelogram or ureteral obstruction at the uretovesical junction could not be ruled out. The general surgery team performed a drain placement shortly after the study to relieve abdominal pressure which had begun to compromise respirations. Over the ensuing 60 minutes, the patient drained $1 \mathrm{~L}$ of fluid with scant output thereafter. Ascitic fluid analysis revealed elevated $\mathrm{Cr}$ level of $3.0 \mathrm{mg} / \mathrm{dL}$. Following the fluid removal, the patient's respiratory status improved rapidly.

On day of life 27, the patient was discharged home with close follow-up by both surgical services, genetics, nephrology, and the plastic surgery, and neurological surgery services. She neither had any further episodes of oliguria, nor any abnormalities in renal function.

\section{Discussion}

There is currently no clear distinction of how OEIS complex occurs during development. Initial hypotheses included failure of mesodermal tissue to migration ${ }^{6}$ and failure of formation of the caudal fold because of the splanchnic and somatic tissue layer failure. ${ }^{7}$ Recent theories focus on malfunction of the umbilical ectodermal placode and primitive streak/caudal eminence in gastrulation although lack of identifiable cause makes prediction of abnormalities difficult. ${ }^{8}$

Exstrophy of the bladder has the most clinically variable presentation and is most commonly noted to be a bisected set of hemibladders. At the bases of these hemibladders, there is typically a single ureteral orifice although this too can be variable. Despite these well documented anatomic variations, there have been no reported cases of acute renal failure in the setting of OEIS complex not related to intrinsic disease. ${ }^{9}$ In our patient, assessments of the genitourinary system for obstruction were negative, thus making the source of renal insult difficult to ascertain, but most importantly not postrenal in initial examinations. The patient's overwhelming ascites on day 5 may have caused a compressive pathology, or Page kidney, to the patient's renal disease but negative retrograde cystourethrogram did not demonstrate a clear postrenal pathology. While traditional Page kidney is noted to be with subcapsular or subcapsular space, we hypothesize that similar pathology could have also been attributed to massive ascitic load. ${ }^{10}$ The patient was treated as if suffering from a prerenal, non-intrinsic insult, and thus was administered large volumes of FFP, pRBCs, and NS. Although the patient was able to produce adequate volumes of urine to remain fluid balanced, the role of third spacing of fluid volume in this clinical vignette clouded the overall picture.
The patient's resolution of renal disease after the first week suggested an acute process; however, no biopsy or confirmatory tests were performed in this patient. A report from 2007 documented a patient with OEIS complex and glomerulocystic renal disease, but this patient had the nonfunctional organ removed, which resolved laboratory abnormalities, unlike our patient who did not require this action. ${ }^{9}$

It is our assessment that this patient may have had elements of prerenal, renal, and postrenal disease during her first week. Secondary to traumatic cord separation, the patient likely initially developed a prerenal azotemia which we believe resulted in an acute kidney injury. This hypothesis explains both lack of persistent renal pathology and clinical response to fluid resuscitation in the first 48 hours. As the patient began developing ascites from third spacing on day 5, we believe this superimposed postrenal pathology on existing acute kidney injury. This later hypothesis is justified by immediate improvements in BUN and $\mathrm{Cr}$ following ascites tap.

\section{Conclusions}

The management of OEIS is complex, and requires an interdisciplinary surgical approach from pediatric urology, pediatric general surgery, and neurosurgery. The driving factor behind surgical corrections is to prevent postrenal obstructive nephropathy, but as defined in our case, renal insults can occur by a variety of mechanisms of which can be prerenal, intrinsic, and postrenal damage. Even after expeditious surgical repair, the ensuing medical management requires meticulous care as exhibited by the multifactorial consideration in managing this patient's renal disease.

\section{Disclosures}

The authors of this article declare neither financial disclosures nor conflict of interest in the creation of this article. The authors declare no support in the means of grants, equipment, or pharmacotherapeutics. No animal or human research was conducted in the production of this article.

\section{References}

1 Carey JC, Greenbaum B, Hall BD. The OEIS complex (omphalocele, exstrophy, imperforate anus, spinal defects). Birth Defects Orig Artic Ser 1978;14(6B):253-263

2 Feldkamp ML, Botto LD, Amar E, et al. Cloacal exstrophy: an epidemiologic study from the International Clearinghouse for Birth Defects Surveillance and Research. Am J Med Genet C Semin Med Genet 2011;157C(4):333-343

3 Hurwitz RS, Manzoni GA, Ransley PG, Stephens FD. Cloacal exstrophy: a report of 34 cases. J Urol 1987;138(4 Pt 2):1060-1064

4 Muecke EC. The role of the cloacal membrane in extrosphy: the first successful experimental study. J Urol 1964;92: 659-667

5 Phillips TM, Salmasi AH, Stec A, Novak TE, Gearhart JP, Mathews RI. Urological outcomes in the omphalocele exstrophy imperforate anus spinal defects (OEIS) complex: experience with 80 patients. J Pediatr Urol 2013;9(3):353-358 
Santoro et al.

6 Mukherjee B, McCauley E, Hanford RB, Aalsma M, Anderson AM. Psychopathology, psychosocial, gender and cognitive outcomes in patients with cloacal exstrophy. J Urol 2007;178(2):630-635, discussion 634-635

7 Duhamel B. Embryology of exomphalos and allied malformations. Arch Dis Child 1963;38(198):142-147

8 van der Putte SCJ, Spliet WGM, Nikkels PG. Common ("classical”) and covered cloacal exstrophy: a histopathological study and a reconstruction of the pathogenesis. Pediatr Dev Pathol 2008; 11(6):430-442

9 Hong R, Lim SC, Jang JW, et al. OEIS complex with glomerulocystic kidney disease: a case report. Pediatr Dev Pathol 2007;10(2): $121-124$

10 McCune TR, Stone WJ, Breyer JA. Page kidney: case report and review of the literature. Am J Kidney Dis 1991;18(5): 593-599 